\title{
The UNAM Scanning Fabry-Perot Interferometer (PUMA) for the Study of the Interstellar Medium
}

\author{
F. Angeles, A. Bernal, F. Cobos, F. Garfias, L. Gutiérrez, R. Langarica, \\ M. Rosado, C. Tejada, S. Tinoco
}

Instituto de Astronomía, UNAM. Ap. Postal 70-264, CP04510 México, D.F.

\begin{abstract}
We describe recent advances in the development of a scanning Fabry-Perot interferometer applied to the study of the kinematics of the interstellar medium at the Observatorio Astronómico Nacional at San Pedro Mártir, B.C. México.
\end{abstract}

\section{General Description}

The kinematics of interstellar matter in cosmic nebulae can be determined by observations at optical wavelengths with the use of a Scanning Fabry-Perot Interferometer (SFPI) with a bidimensional detector. This combination allows a wider field of view and a higher spatial and spectral resolution than those obtained with a classical high-dispersion spectrograph. The system called PUMA is an instrument consisting of a focal reducer coupled to a SFPI, which has been developed for the Observatorio Astronómico Nacional at San Pedro Mártir, B.C., México. It has a series of interference filters and a calibration system consisting of three discharge lamps and a diffuser. The SFPI can be moved out of the optical path in order to acquire direct images. It will be installed at the 2.0 m. telescope in its $\mathrm{f} / 7.9$ Ritchey-Chretien focus. The images produced by this instrument will be focused on an optoelectronic detector, a CCD, or a Mepsicron, depending on the spectral range used. The PUMA will provide: a) direct imagery with interference or wide-band filters. b) interferograms at $\mathrm{H} \alpha,[\mathrm{NII}](\lambda$ $6584 \mathrm{~A})[\mathrm{OIII}](\lambda 5007 \mathrm{~A}),[\mathrm{SII}](\lambda 6717 \mathrm{~A})$.

\section{Optical System}

This system will cover a field of $10^{\prime}$ and will provide a reducing factor of 2 in the telescope focal ratio ( from $\mathrm{f} / 7.9$ to $\mathrm{f} / 3.95$ ). The plate scale will be of $0.6^{\prime \prime}$ per pixel for the $1024 \times 1024$ Thomson CCD in work at the observatory. The focal reducer consists of a collimator and a camera. The collimator is made up of two different optical components: a doublet and a triplet. Both were polished from PSK3 and FK54 optical glasses. The selection of materials was based on a design of Marseilles Observatory to achieve an apochromatic system, covering the desired wide spectral range, from the ultraviolet $(\lambda 3650 \AA)$ to the infrared $(\lambda$ $8650 \AA$ ). An important characteristic of this system is that the collimated light bundle and the instrument configuration will allow the integration of optical 
elements such as multilens arrays, prisms or grisms. This will make easy to use it as a tool for other kind of studies. The camera used is a commercial Leitz lens, however, we are constructing a camera suitable for the wide spectral range covered by the collimator.

\section{Mechanical System}

The mechanical system consists of an aluminum structure to attach the instrument to the telescope. The PUMA includes movable parts like: a carousel with three calibration lamps, a wheel with seven filters and a rail for moving the SFPI out of the optical path. Tolerances in the flexure of the mechanical structure are defined by half the size of the detector pixels. In order to have a stiff structure we decided to include a flat mirror to deviate the optical path 60 degrees from the optical axis of the the telescope. Besides, the structural design uses aluminum tubes for greater stiffness with a minimum weight.

\section{The Interferometer}

The SFPI used is a 2 inches Queensgate servo-stabilized by means of the CS100 Queensgate system. The CS100 allows for the adjustment of the servosystem parameters, the parallelism, and the separation between plates with a response time of 0.5 seconds in steps of $0.5 \mathrm{~nm}$. Recent laboratory test to this interferometer have allowed us to determine an effective "Finesse" of about 30. The order of interference is 355 , the free spectral range of $18.48 \mathrm{~A}$ and the sampling spectral resolution of $0.31 \mathrm{~A}$ at $\mathrm{H} \alpha$.

\section{Electronic Control}

All the functions of the CS100 are achieved through a control bus that allows for its complete computerized remote control operation. Both the instrument and the etalon will be controlled with an Octagon microcomputer, model $\mu \mathrm{PC}$ 5080 , based on an 12 bit 64180 processor (Z80 code compatible) running at 9.216 MHz.The software consists of an operative system and a Basic compiler designed for control applications named CAMBASIC. All the system will be supervised by a SPARC workstation as the host computer. The image series corresponding to different wavelengths will be stored in its magnetic memory. 